\title{
PReS-FINAL-2261: Prevalence of orofacial symptoms and signs in patients with juvenile fibromyalgia
}

\author{
C Len", L Zwir, M Fraga, MT Terreri \\ From 20th Pediatric Rheumatology European Society (PReS) Congress \\ Ljubljana, Slovenia. 25-29 September 2013
}

\section{Introduction}

Fibromyalgia may coexist with other clinical conditions such temporomandibular disorders (TMD). Temporomandibular disorder is a term embracing clinical disorders that involve the masticatory musculature, the temporomandibular joints, and associated structures.

\section{Objectives}

The purpose of this study was to assess the prevalence of orofacial symptoms and signs in patients with juvenile fibromyalgia.

\section{Methods}

Twenty-eight consecutive patients (22 girls) who presented to our outpatient pediatric rheumatology clinic and fulfilled the ACR criteria of fibromyalgia were included in this study. All patients underwent a rheumatologic examination performed by a pediatric rheumatologist, and an orofacial examination performed by a single dentist at the same data. The patients were interviewed according to a standardized questionnaire concerning the presence of orofacial pain and functional impairment and were submitted to a clinical evaluation following a structured protocol.

\section{Results}

The mean age at the evaluation was 13 years (range 8-18 years) and the mean follow-up time was 3.3 years ( 0.3 to 12). Orofacial symptoms were reported in 14 (50\%) of patients. The most common subjective symptoms were impaired ability with chewing (50\%), pain in function (28.6\%), and pain at rest (17.8\%). We found that the vast majority of our patients (89.3\%)reported pain on palpation in at least two of six sites in the orofacial region, but only 7 (25\%) had pain during mandibular movements, and nobody had restrictions to open their mouths $40 \mathrm{~mm}$ or more.

\section{Conclusion}

Although palpation of the orofacial region is not included in the diagnostic criteria for fibromyalgia, a large number of juvenile patients presented with pain on palpation in this region. This study suggests the need for interdisciplinary strategies to effectively diagnose and treat this chronic condition.

\section{Disclosure of interest}

None declared.

Published: 5 December 2013

\section{doi:10.1186/1546-0096-11-S2-P251}

Cite this article as: Len et al.: PReS-FINAL-2261: Prevalence of orofacial symptoms and signs in patients with juvenile fibromyalgia. Pediatric Rheumatology 2013 11(Suppl 2):P251. 